\title{
Endothelial GC-A can be a therapeutic target for metabolic syndrome
}

\author{
Takeshi Tokudome ${ }^{1 *}$, Kentaro Otani ${ }^{2}$, Ichiro Kishimoto ${ }^{1}$, Yuanjie Mao ${ }^{1}$, Kazuwa Nakao ${ }^{3}$, Kenji Kangawa ${ }^{4}$ \\ From 6th International Conference on cGMP: Generators, Effectors and Therapeutic Implications \\ Erfurt, Germany. 28-30 June 2013
}

\section{Background}

Atrial natriuretic peptide (ANP) has been used clinically for the treatment of heart failure patients in Japan, and also exhibits a variety of physiological effects through binding guanylyl cyclase-A (GC-A) receptor. We and other groups has been reported that GC-A is abundantly expressed in endothelial cells. In the present study, we explored the therapeutic potential of endothelial ANP/ GC-A system for the treatment of metabolic syndrome.

\section{Methods}

We generated endothelial cell-specific GC-A transgenic mice using Tie2 promoter and enhancer (EC-GC-A-Tg), inducible endothelial cell-specific GC-A transgenic mice (Inducible EC-GC-A-Tg), and also endothelial cell-specific GC-A knockout mice (EC-GC-A-KO). In addition, we used eNOS transgenic mice (eNOS-Tg). For the evaluation of blood pressure, telemetry system and tail-cuff method were used. Insulin resistance was evaluated by intra-peritoneal glucose tolerance test (IPGTT) and intra-peritoneal insulin tolerance test (IPITT).

\section{Results}

The phenotypes of EC-GC-A-Tg were very unique. Systolic blood pressure in EC-GC-A-Tg was significantly lower compared with wild-type mice (WT). In addition, heat weight/body weight ratio and arterial elastance were smaller and lower in EC-GC-A-Tg compared with WT. Synchrotron radiation angiography showed decrease of basal vascular tone in EC-GC-A-Tg and increase in ECGC-A-KO compared with in each control mice. Basal body weight of WT and EC-GC-A-Tg, flox mice and ECGC-A-KO were comparable. However, after taking
8 weeks of high-fat diet, increase of body weight of ECGC-A-Tg was significantly less than WT. On the other hand, EC-GC-A-KO showed more than increase of body weight. IPGTT and IPITT showed improvement of insulin tolerance in EC-GC-A-Tg, and worsen in EC-GC-A$\mathrm{KO}$ compared with in each control mice. Interestingly, Inducible EC-GC-A-Tg showed less increase of body weight accompanied by aging. The body weight of eNOS-Tg was comparable with WT before and after taking 8 weeks of high-fat diet.

\section{Conclusion}

These data suggest that endothelial ANP/GC-A system can be a therapeutic target for metabolic syndrome.

\section{Authors' details}

'Department of Biochemistry, National Cerebral and Cardiovascular Center Research Institute, Osaka, Japan. ${ }^{2}$ Department of Regenerative Medicine and Tissue Engineering, National Cerebral and Cardiovascular Center Research Institute, Osaka, Japan. ${ }^{3}$ Department of Medicine and Clinical Science, Kyoto University Graduate School of Medicine, Kyoto, Japan. ${ }^{4}$ National Cerebral and Cardiovascular Center Research Institute, Osaka, Japan.

Published: 29 August 2013

doi:10.1186/2050-6511-14-S1-P73

Cite this article as: Tokudome et al.: Endothelial GC-A can be a

therapeutic target for metabolic syndrome. BMC Pharmacology and Toxicology 2013 14(Suppl 1):P73.

\footnotetext{
* Correspondence: tokudome@ncvc.go.jp

'Department of Biochemistry, National Cerebral and Cardiovascular Center Research Institute, Osaka, Japan

Full list of author information is available at the end of the article
}

(c) 2013 Tokudome et al; licensee BioMed Central Ltd. This is an Open Access article distributed under the terms of the Creative 